\title{
GESTIÓN DE ZONAS VERDES URBANAS Y PERIURBANAS PARA LA CONSERVACIÓN DE LA BIODIVERSIDAD: EL CASO DE VITORIA-GASTEIZ
}

\author{
Fernando de Juana Aranzana
}

Unidad de Anillo Verde y Biodiversidad. Ayuntamiento de Vitoria-Gasteiz Palacio Zulueta Paseo de la Senda 2. 01007-VITORIA-GASTEIZ (España). Correo electrónico: fdejuana@ vitoria-gasteiz.org

\begin{abstract}
Resumen
Los espacios verdes urbanos y periurbanos pueden jugar un papel importante en la conservación de la biodiversidad. Entre los factores que condicionan la abundancia y diversidad de la fauna de los parques urbanos, los más relevantes son el tamaño, la heterogeneidad del hábitat, la edad del parque y las molestias de origen antrópico. El ejemplo de Vitoria-Gasteiz confirma la mayor importancia para la conservación de la biodiversidad de las zonas verdes periurbanas en comparación con las urbanas. Los parques y jardines del interior de la ciudad, en general de pequeño tamaño y con una cubierta vegetal poco diversificada, presentan una escasa riqueza faunística. En el caso de las zonas verdes periurbanas son varios los factores que contribuyen a su elevada tasa de biodiversidad: su mayor superficie, su localización exterior al casco urbano, su densa cubierta vegetal y elevada diversidad de hábitats, y la baja densidad de su red de caminos. Por otro lado, los parques del Anillo Verde de Vitoria-Gasteiz son objeto de una gestión específicamente dirigida a la conservación de la biodiversidad. El mantenimiento diferenciado de céspedes y praderas y la instalación de nidales y refugios artificiales pueden citarse a este respecto como prácticas particularmente interesantes.
\end{abstract}

Palabras clave: Flora, fauna, Aves, Anillo Verde, Salburua

\section{LA BIODIVERSIDAD URBANA}

El mantenimiento de la diversidad biológica o biodiversidad fue incorporado en la conferencia de Río de Janeiro de 1992 como uno de los grandes objetivos de las estrategias mundiales de conservación. Desde entonces, las investigaciones en este campo han revelado que no sólo las áreas naturales y semi-naturales pueden tener una alta diversidad de flora, fauna y hábitats, sino que también las zonas urbanas y periurbanas pueden atesorar una amplia variedad de organismos y comunidades (CELECIA, 1997). Hay que tener en cuenta que una alta proporción de zonas urbanas se sitúan en fondos de valle y sistemas riparios altamente productivos, que poseen potencialmente una gran riqueza florística y faunística. De hecho, a gran escala existe una correlación positiva entre la población humana y el número de especies de plantas y vertebrados presentes en una región, lo que significa que los seres humanos compiten con la biodiversidad por el espacio vital (PAUTASSO, 2007). En consecuencia, las reservas naturales en la proximidad de las ciudades pueden albergar una gran diversidad de especies, ya que estas áreas protegidas son con frecuencia fragmentos de ecosistemas con una alta biodiversidad (SCHAEFER, 1994).

A escalas intermedias se ha constatado que los ecosistemas urbanizados pueden ser más ricos 
en especies que sus inmediaciones, al presentar un mosaico más variado de medios y microhábitats: parques, jardines, céspedes, huertos, setos, solares, eriales, cursos de agua... (PAUTASso, 2007). Es el caso de algunas ciudades de China y Nueva Zelanda, por ejemplo, en las que se ha registrado una mayor riqueza y diversidad florística que en el área rural circundante (AlveY, 2006). En áreas muy humanizadas las zonas verdes urbanas y periurbanas pueden contener niveles comparativamente altos de biodiversidad, como en Flandes, donde en 15 parques están representadas el 30\% de las especies de plantas silvestres, el $40 \%$ de las mariposas, el $50 \%$ de las aves reproductoras y el $60 \%$ de los anfibios de la región (CORNELIS \& HERMY, 2004).

El papel de las zonas verdes urbanas y periurbanas puede ser también importante en la conservación de especies amenazadas. Resulta sorprendente, por ejemplo, que el municipio de Estocolmo, con una alta densidad de población, albergue dos tercios de las especies de la Lista Roja de Suecia (Colding et al., 2003).

Los estudios de pequeña escala coinciden sin embargo en que la urbanización produce en general una simplificación de los hábitats y un empobrecimiento de las comunidades animales y vegetales (PAUTASSO, 2007; MCKINNEY, 2008). No obstante, niveles moderados de urbanización pueden incrementar en algunos casos la riqueza específica. En un estudio desarrollado en Berlín, por ejemplo, el número más alto de especies vegetales por kilómetro cuadrado se encontró en la zona de transición entre el centro y las afueras, donde el mosaico de usos del suelo era más heterogéneo (ZERBE et al., 2002). Análogamente, al estudiar la distribución y abundancia de las aves nidificantes a lo largo de un gradiente urbano en California, se observaron máximos de riqueza específica, diversidad y densidad en las zonas periurbanas moderadamente transformadas, si bien el incremento del número de especies resultaba de la presencia en estas zonas de especies ampliamente distribuidas, a costa de las especies propias del hábitat original (BLAIR, 1996). Un patrón similar se encontró para la comunidad de mariposas a lo largo del mismo gradiente (BLAIR, 1999).

Parece, por tanto, que niveles intermedios de transformación humana pueden afectar positivamente tanto a la diversidad del hábitat como a la riqueza y a la diversidad total de especies. En el caso de las aves, sin duda el grupo mejor estudiado, se ha comprobado que la mayor heterogeneidad del medio aumenta la diversidad y la abundancia de los recursos disponibles, mientras que un alto grado de urbanización tiene el efecto contrario, al reducir de manera sustancial la producción primaria (BLAIR, 1996). Además, una cierta fragmentación de un hábitat anteriormente continuo incrementa el ecotono, que se reduce sin embargo a medida que el desarrollo urbano tiende a eliminar el hábitat natural (GORDON \& Forman, 1983). Todo ello explica que las zonas verdes periurbanas tengan comparativamente una importancia mucho mayor para la conservación de la biodiversidad que los parques y jardines urbanos.

La ocupación por la fauna de las zonas verdes en el interior de las ciudades viene también condicionada por la distinta capacidad dispersiva de cada especie. La disminución de la riqueza y diversidad de las comunidades de carábidos en retazos forestales urbanos en Rennes respecto de los bosques periurbanos se explicó por el efecto negativo de las carreteras y otras superficies construidas sobre la dispersión de estos escarabajos, mientras que la estructura de las comunidades de aves no parecía afectada en este caso por la fragmentación del paisaje urbano (CROCI et al., 2008). Sin embargo, otros estudios han concluido que también las aves pueden ser reacias a atravesar grandes discontinuidades, de manera que la falta de conectividad puede constituir un problema en zonas verdes altamente fragmentadas por la trama urbana (DESROCHERS \& HANNON, 1997; SAVARD et al., 2000).

Diversos estudios, la mayoría de ellos centrados en las aves, han tratado de determinar los factores que condicionan la abundancia y diversidad de la fauna de los parques urbanos. A este respecto el tamaño es el factor principal a la hora de explicar la riqueza específica, debido a que cuanto mayor es el parque mayor es el número de especies que pueden ver satisfechas en él sus necesidades espaciales mínimas (FERNÁNDEZ-JURICIC \& JOKIMÄKI, 2001) (Figura 1). Desde el punto de vista de la biodiversidad, por tanto, siempre será preferible un parque grande que muchos parques pequeños, ya que los parques grandes albergan todas las especies 


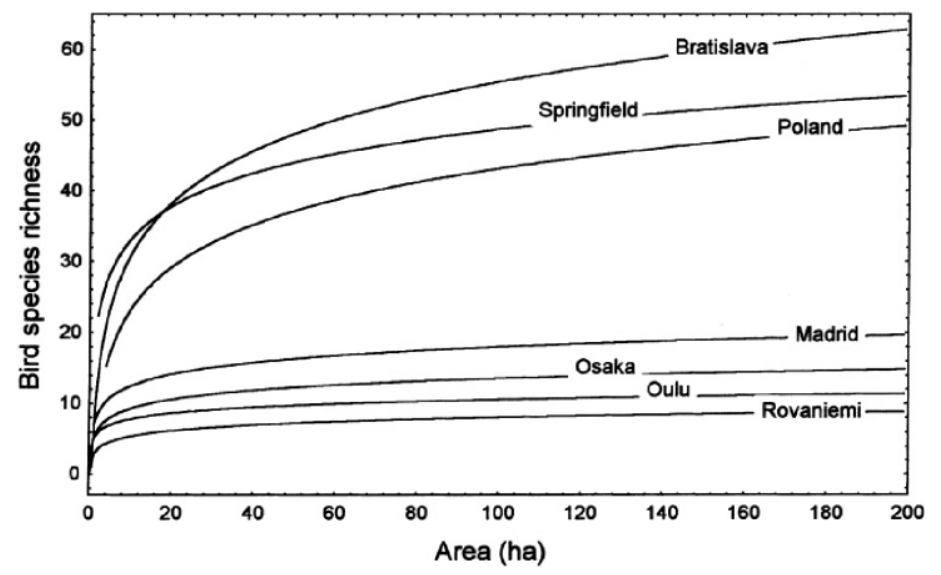

Figura 1. Relación entre la superficie de los parques urbanos y la riqueza de su comunidad ornítica en diferentes ciudades (tomado de FERNÁNDEZ-JURICIC \& JOKIMÄKI, 2001)

presentes en los pequeños más algunas que sólo se encuentran en los grandes.

La diversidad y la densidad de la vegetación y la heterogeneidad del hábitat son también factores que incrementan la biodiversidad de las zonas verdes. Así, en los parques urbanos de Madrid se comprobó que la relación negativa entre el número de especies de aves y la proporción de superficie pavimentada de los parques era muy significativa (FERNÁNDEZ-JURICIC \& JOKIMÄKI) (Figura 2). A medida que se reduce la cubierta vegetal, la comu- nidad aviar se simplifica y tiende a estar dominada por unas pocas especies bien adaptadas al medio urbano. La existencia de cursos de agua, grandes coníferas y arbustos productores de bayas son variables que explicaron en gran parte la presencia de diferentes especies en las zonas verdes de Vancouver (MELLES et al., 2003).

La flora de los jardines y parques urbanos suele estar dominada por especies exóticas, pero una alta proporción de estas especies puede interactuar potencialmente con los herbívoros nativos,

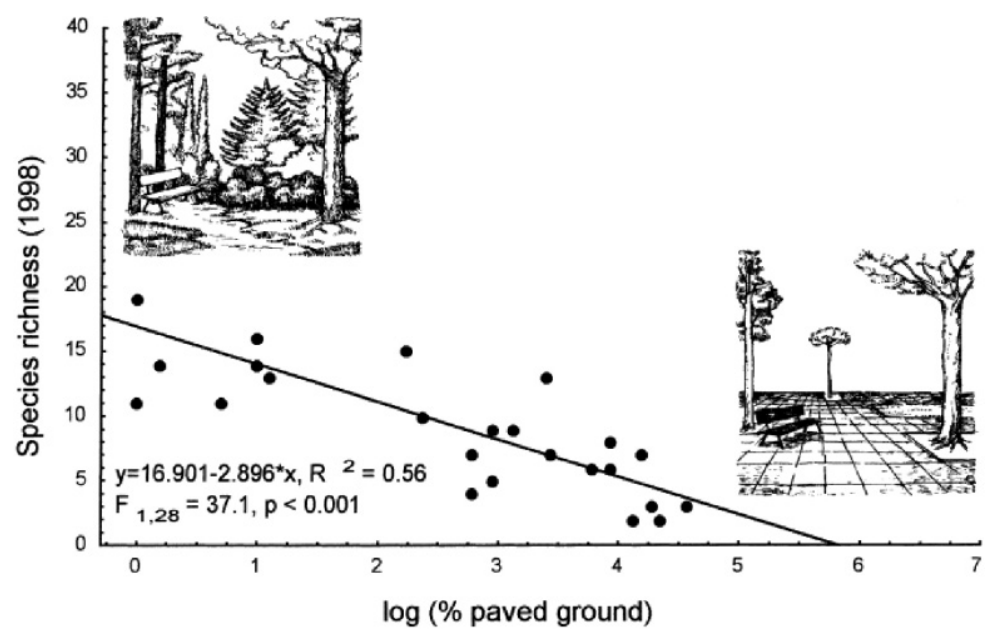

Figura 2. Relación entre la superficie pavimentada y la riqueza de su comunidad ornítica en los parques urbanos de Madrid (tomado de FERNÁNDEZ-JURICIC \& JOKIMÄKI, 2001) 
constituyendo importantes fuentes de frutos, polen y néctar (SMiтH et al., 2006). Para algunos insectos las plantas exóticas constituyen huéspedes alternativos que pueden soportar poblaciones mayores que las existentes en los hábitats naturales, donde las plantas huéspedes autóctonas son escasas (CONNOR et al., 2002). Sin embargo, la proporción de especies animales autóctonas está positivamente correlacionada con el volumen y la diversidad de la vegetación autóctona, por lo que la utilización en jardinería de especies de plantas locales se ha propuesto como una forma efectiva de conservación de la biodiversidad (MCKINNEY, 2002).

En Rennes, la riqueza específica de la comunidad de micromamíferos resultó estar positivamente correlacionada con la riqueza, diversidad y cobertura del estrato herbáceo (CROCI et al., 2008). El desarrollo y composición de las comunidades herbáceas de las zonas verdes están a su vez fuertemente condicionados por el régimen de siegas. HELDEN \& LEATHER (2004) comprobaron que tanto la riqueza específica como la abundancia de hemípteros en rotondas urbanas eran mayores cuanto menor era la frecuencia de corte de la hierba (Figura 3).

El efecto negativo para la biodiversidad de los manejos intensivos fue constatado también por Donvan et $a l$. (2005) en Birmingham, donde cuatro parques sometidos a una fuerte intervención humana reunían en conjunto un $18 \%$ del total de las especies de insectos inventariadas,

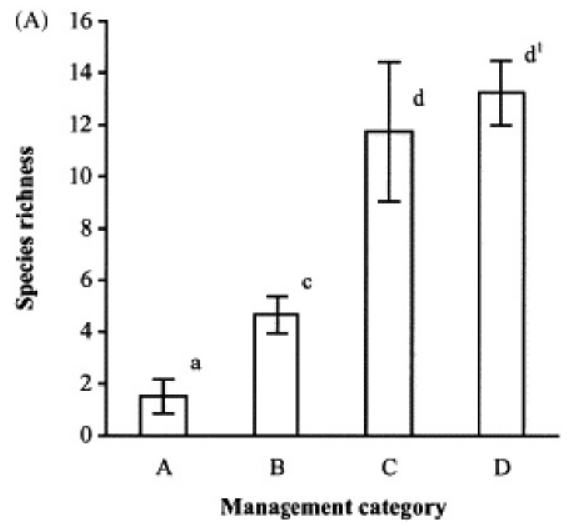

mientras que en cuatro solares abandonados esta cifra alcanzaba el $82 \%$.

La edad de los parques se sabe que tiende a incrementar el número de especies presentes en ellos, debido a que el progresivo desarrollo de la vegetación favorece una mayor complejidad del hábitat, lo que permite la colonización por nuevas especies que ocupan nichos inexistentes en las zonas verdes de reciente creación (HoHTOLA, 1978). La edad del arbolado también está correlacionada positivamente con la riqueza de la comunidad aviar (STROHBACH et al., 2009).

La avifauna de los parques urbanos parece asimismo estar relacionada con la que ocupa el entorno próximo de la ciudad, debido por una parte a que es más probable que los individuos que se dispersan se asienten cerca de su área natal y, por otra, a que las especies pueden suplementar los recursos de los parques urbanos con los disponibles en las zonas periurbanas (SAVARD et al., 2000; MELLES et al., 2003). RUDD et al. (2002) han destacado la importancia de los corredores ecológicos cuando conectan grandes zonas verdes ricas en biodiversidad con parques más pequeños que dependen total o parcialmente de la inmigración de individuos procedentes de aquéllas. El aumento de la complejidad de la cubierta vegetal en el viario urbano, especialmente en grandes avenidas, constituye a este respecto una potente herramienta para mejorar la conectividad de las zonas verdes (FERNÁNDEZJURICIC \& JOKIMÄKI, 2001).

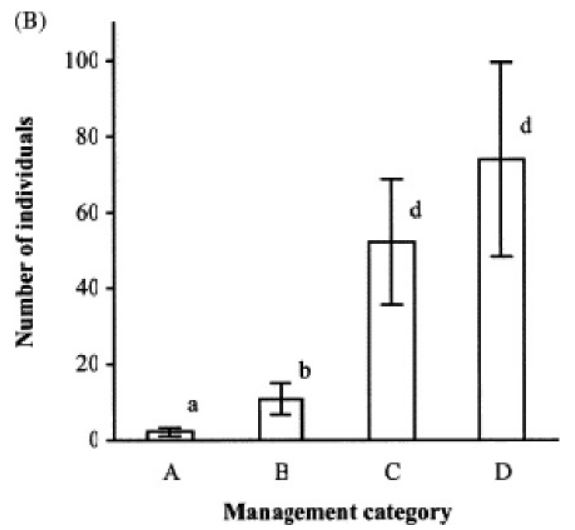

Figura 3. Riqueza media (A) y abundancia media (B) de hemípteros en herbazales sometidos a distinta frecuencia de siegas: A, siegas cada 7 días; $B$, siegas cada 14 días; $C$, siegas cada 40 días; $D$, una siega o menos al año (tomado de HELDEN \& LEATHER, 2004) 
La biodiversidad urbana está finalmente condicionada por las molestias de origen antrópico. Se ha comprobado que la actividad recreativa afecta negativamente a la riqueza de especies de aves y que la densidad de las poblaciones reproductoras se reduce significativamente en parques muy visitados (FERNÁNDEZ-JURICIC, 2000). En los grandes parques madrileños las especies más tolerantes con la presencia humana, como los gorriones y las palomas domésticas, son más comunes en los bordes, donde la contaminación acústica debida al tráfico y a los peatones es mayor, mientras que las especies menos tolerantes se encuentran principalmente en el interior (FERNÁNDEZ-JURICIC \& JOKIMÄKI, 2001). Una menor relación perímetro/superficie favorecería por tanto una mayor biodiversidad de las zonas verdes, al igual que una menor densidad de caminos interiores.

Las zonas urbanas constituyen un hábitat favorable para el establecimiento de especies exóticas, bien proporcionando recursos tróficos, bien reduciendo la amenaza de sus enemigos naturales o bien alterando las condiciones ambientales, debido por ejemplo al efecto de isla de calor (MCKINNEY, 2006). Además, las especies directa o indirectamente importadas en un área a consecuencia de la actividad humana frecuentemente comienzan su dispersión en las ciudades y por tanto aparecen en ellas con más frecuencia (Zerbe et al., 2002). Estas especies compiten en ocasiones con las autóctonas, pudiendo provocar una reducción de sus poblaciones e incluso extinciones locales (ALVEY, 2006). Por ello, los planes de control y erradicación de especies exóticas invasoras tienen una especial importancia en las zonas urbanas.

\section{CONSERVACIÓN DE LA BIODIVERSIDAD EN VITORIA-GASTEIZ}

La trama verde de la ciudad de VitoriaGasteiz se compone de 445 hectáreas de zonas verdes urbanas (parques, jardines, zonas deportivas, cementerios, bulevares y paseos arbolados) y 660 hectáreas de zonas verdes periurbanas (los 5 grandes parques del Anillo Verde y los corredores que los conectan entre sí).

La única información que poseemos sobre la biodiversidad de las zonas verdes urbanas de
Vitoria-Gasteiz procede de un estudio sobre la avifauna de 20 parques y zonas ajardinadas, en el que se detectaron un total de 13 especies reproductoras, cifra que resulta bastante inferior a la obtenida en otros trabajos similares en escenarios urbanos (DE LA HeRA et al., 2009). La diferente riqueza específica de cada parque, que varió entre 2 y 12, se explicaba en gran medida en función de su superficie y del porcentaje de la cobertura del arbolado. No se obtuvo en cambio una relación significativa entre la riqueza y la cobertura de arbustos, lo que según los autores se explica porque los parques que poseen una mayor proporción de superficie cubierta de arbustos eran los más pequeños, y porque los macizos arbustivos estaban situados preferentemente en la periferia de los parques, donde su contribución a la diversidad de especies podía verse contrarrestada por el efecto borde. Esto, junto con el pequeño tamaño de las zonas verdes estudiadas -entre $0,75 \mathrm{y}$ 19,42 hectáreas - podría explicar el reducido número de especies presentes.

La escasa riqueza de la comunidad ornítica de los parques urbanos vitorianos contrasta notablemente con la correspondiente al parque periurbano de Salburua, sin duda el de mayor valor ecológico de los que integran el Anillo Verde de la ciudad, donde cada año se reproducen entre 63 y 73 especies de aves. Con una superficie de 206 hectáreas, la cubierta vegetal de Salburua está compuesta por un mosaico de pequeños bosquetes y arboledas, repoblaciones forestales, setos y riberas, herbazales y vegetación palustre. Aproximadamente un $20 \%$ de las especies reproductoras -entre 12 y 17 según años - pueden considerarse aves acuáticas, cuya presencia está ligada al sistema de humedales que alberga el parque. En el gráfico de la Figura 4 puede observarse cómo tanto el número de especies como el número de parejas de aves acuáticas nidificantes aumentó significativamente tras las obras de restauración ejecutadas en 1998 y 1999, que multiplicaron aproximadamente por cuatro la superficie de zonas húmedas.

La importancia de la biodiversidad que alberga el parque de Salburua ha podido constatarse al estudiar otros grupos faunísticos. Así, ORTUÑO Y MARCOS (1997) encontraron un total de 108 especies de escarabajos carábidos, varias de ellas de notable interés biogeográfico, en las 44 hectá- 


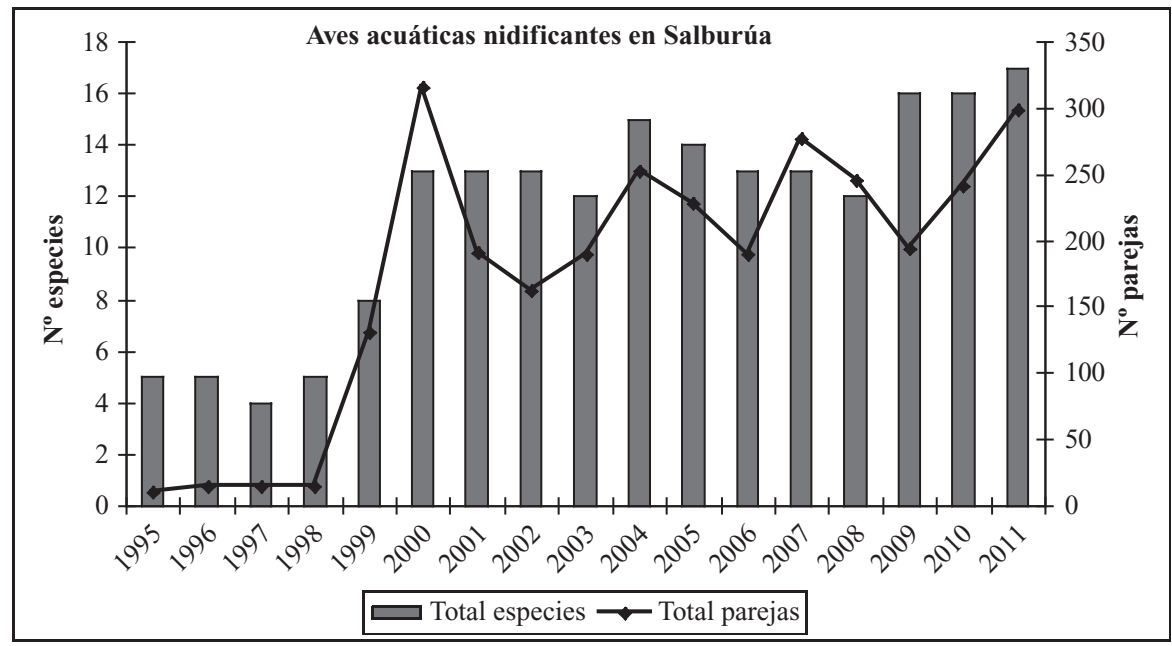

Figura 4. Evolución del número de especies y del número de parejas de aves acuáticas nidificantes en el parque de Salburua

reas de humedales que habían sobrevivido al proceso de desecación iniciado a mediados del siglo XIX, lo que representa aproximadamente un $10 \%$ de la carabidofauna ibérica y un $40 \%$ de la de la Comunidad Autónoma del País Vasco. A juicio de los autores de este estudio no se conocía con anterioridad ningún humedal subsalino que reuniese tan interesante conjunto de especies palustres. Son destacables también las 33 especies de odonatos inventariadas en Salburua, de un total de 76 conocidas para la Península Ibérica, entre las que sobresale por su grado de amenaza Coenagrion mercuriale (VALLADARES, 2010). Otras especies de insectos incluidas como ésta en el Anexo II de la Directiva Hábitats y también presentes en Salburua son la mariposa Euphydryas aurinea y los coleópteros Lucanus cervus y Rosalia alpina.

Entre los vertebrados es destacable asimismo la presencia de varias especies amenazadas, en particular el Visón Europeo (Mustela lutreola) y la Rana Ágil (Rana dalmatina). La sedimentación regular de Carricerín Cejudo (Acrocephalus paludicola) durante la migración postnupcial ha justificado la catalogación de este espacio como Área Importante para la Conservación de las Aves (IBA). Además, los humedales de Salburua están incluidos en el Convenio Ramsar y han sido declarados Lugar de Importancia Comunitaria dentro de la Red Natura 2000.
El elevado valor natural de Salburua se debe probablemente en gran medida a que los trabajos de acondicionamiento del parque, que comenzaron en 1994, respetaron escrupulosamente la vegetación natural existente, favoreciéndose además su regeneración espontánea en extensas superficies anteriormente dedicadas al cultivo agrícola. Entre los hábitats representados en este espacio hay 6 de interés comunitario (ríos con vegetación leñosa de Salix eleagnos, formaciones de grandes cárices, pastos mesófilos sobre sustratos calcáreos, prados de hierbas altas y juncos, robledales y bosques de galería), que ocupan en conjunto el $47 \%$ de su superficie total.

El caso de otros parques del Anillo Verde es bien distinto. En el de Olárizu, por ejemplo, un extenso pastizal caracterizado por un alto grado de hidromorfía fue drenado en 1984 para favorecer el uso recreativo, ajardinándose luego con césped y arbolado ornamental. Subsisten sin embargo algunos vestigios de los trampales y prados-juncales originales, en los que se han inventariado hasta la fecha 108 especies vegetales, frente a las 51 de las praderas y céspedes de origen artificial. En este parque se herborizaron en 1983 dos especies incluidas en el Catálogo de Especies Amenazadas del País Vasco, Galium boreale y Senecio carpetanus, que no habían vuelto a observarse desde entonces (URIBEECHEVARRÍA, 2010), hasta que en 2011 se redes- 
cubrió una pequeña población de la primera de ellas (AGUT, 2012). Además, los prados-juncales de Olárizu están considerados como hábitat de interés comunitario y los trampales como hábitat prioritario por su riqueza en orquídeas.

En los parques de Zabalgana y Armentia, que también forman parte del Anillo Verde, las poblaciones de orquídeas han sido favorecidas por una gestión diferenciada dirigida a permitir la floración de estas especies. El retraso progresivo de la primera siega ha incrementado año tras año el número de especies de orquídeas que llegan a florecer, alcanzándose en 2012 las 24 especies, una de ellas, Himantoglossum hircinum, considerada de interés especial en el Catálogo de Especies Amenazadas del País Vasco. La disminución de la frecuencia de las siegas favorece además como hemos visto la riqueza y abundancia de insectos herbívoros y, en consecuencia, de sus predadores. En algunos casos se ha optado incluso por la no-gestión, permitiendo la evolución de las comunidades herbáceas hacia estados más avanzados dentro del proceso de sucesión vegetal (GoNZÁLEZ Y GONZÁLEZ, 2007).

Durante los años 2008 y 2009 se llevaron a cabo en el parque de Salburua una serie de actuaciones dirigidas al incremento de la biodiversidad, dentro del llamado proyecto INBIOS (LOBO, 2008). Entre las medidas orientadas a la mejora de las poblaciones de especies amenazadas, cabe destacar la colocación de 95 cajas-refugio para murciélagos, de las cuales se ocuparon 6 en 2010 y 40 en 2011 por 3 especies distintas; una de ellas, el nóctulo pequeño (Nyctalus leisleri), no había sido detectado hasta la fecha en el parque, lo que viene a constatar que la ausencia de refugios naturales (cuevas, árboles viejos) o artificiales (edificios antiguos) puede estar condicionando el asentamiento de algunas especies de quirópteros (AlCALDE Y MARTínEZ, 2011). Análogamente, la construcción de una colonia artificial de Avión Zapador (Riparia riparia), especie considerada "vulnerable" en el Catálogo Vasco de Especies Amenazadas, ha permitido la nidificación de 8 parejas en 2013.

El proyecto INBIOS contempló igualmente algunas intervenciones dirigidas a la mejora del estado de conservación de los hábitats de Salburua, concretamente el decapado de los vasos lagunares para reducir el depósito de materia orgánica y favorecer el desarrollo de la vegetación acuática, y la siega con retirada de restos de praderas y juncales para el rejuvenecimiento de estas comunidades herbáceas.

En Salburua se llevan a cabo regularmente acciones de control de especies exóticas invasoras, como el Visón Americano (Neovison vison) y diversas especies de peces y galápagos, que representan una amenaza para la conservación de algunas especies autóctonas (LOBO, 2008). Se han ejecutado además otras medidas como la creación de pequeñas lagunas estacionales libres de peces.

Otras medidas aplicadas en los parques del Anillo Verde son por ejemplo el apilado de madera muerta para favorecer a los insectos xilófagos o el respeto a las épocas críticas para la reproducción de algunas especies como el visón europeo.

Los parques del Anillo Verde de Vitoria-Gasteiz, debido a su situación limítrofe con el casco urbano, soportan una elevada presión de uso público, estimada en 2012 en 1,6 millones de visitas. Debido a que una gran mayoría de los visitantes no abandonan en sus recorridos los caminos afirmados, el trazado de la red viaria tiene una importante repercusión en el impacto que la presencia humana puede tener en la fauna silvestre. En el parque de Salburua los itinerarios peatonales bordean las dos lagunas principales, dejando en su interior sendas superficies de 78 y 33 hectáreas. En la mayor de ellas el acceso del público está limitado además por un vallado cinegético.

Las labores de divulgación y sensibilización en temáticas vinculadas a la conservación de la biodiversidad en los parques del Anillo Verde son muy intensas, destacando en esta labor el Centro de Interpretación Ataria (http://www.vitoria-gasteiz.org/we001/was/we001Action.do?idioma=es \&accionWe001=ficha\&accion=ataria) ubicado en Salburua. Estas actuaciones contribuyen claramente a generar una mayor sensibilidad social y una mejor acogida de las necesarias inversiones públicas vinculadas a la promoción y conservación de la biodiversidad.

Por último, en todos los parques del Anillo Verde se realiza una intensa labor de monitorización de parámetros ecológicos clave de cara a testar la efectividad de las labores de restauración ambiental acometidas en ellos y sus manejos posteriores. Estos seguimientos científicos son objeto de una programación a largo plazo y sus 
resultados han contribuido a lo largo de los años a promover figuras de conservación de algunos espacios, a reorientar patrones de gestión en los parques, a aplicar medidas concretas de protección de algunas especies o hábitats amenazados o a acceder a ayudas financieras vinculadas a la correcta preservación de la biodiversidad.

\section{CONCLUSIONES}

El ejemplo de Vitoria-Gasteiz confirma la mayor importancia para la conservación de la biodiversidad de las zonas verdes periurbanas en comparación con las urbanas. La escasa riqueza de la fauna presente en los parques y jardines del interior de la ciudad parece obedecer principalmente a su pequeño tamaño -menor de 20 hectáreas-y a la falta de complejidad en la estructura de su cubierta vegetal. La alta frecuencia de las siegas, la reducida utilización de especies arbustivas y la ausencia en general de arbolado viejo, madera muerta, refugios o puntos de agua se traduce en medios muy homogéneos y con comunidades vegetales y animales muy empobrecidas.

En el caso de las zonas verdes periurbanas son varios los factores que contribuyen a su elevada tasa de biodiversidad. En primer lugar su mayor superficie -entre 60 y 200 hectáreas-; en segundo su localización exterior al casco urbano, que reduce por un lado algunas molestias como el ruido o la iluminación artificial y favorece por otro su conectividad ecológica con otras zonas naturales; en tercer lugar su densa cubierta vegetal y elevada diversidad de hábitats, resultado en unos casos de la conservación de la vegetación original y en otros de un proceso de recuperación y manejo posterior con criterios ecológicos; finalmente, la baja densidad de la red de caminos limita en buena medida el impacto negativo de la afluencia de público.

El modelo convencional de diseño y gestión de zonas verdes, guiado por criterios estéticos y predominantemente orientado al uso de los seres humanos, no es adecuado para satisfacer los requerimientos de la biodiversidad urbana. El primer objetivo a este respecto debe ser el de evitar la uniformidad tanto en la concepción como en el manejo de las zonas verdes. Los mantenimientos menos intensivos, además de reducir los costes de gestión, favorecen la diversificación de las comunidades florísticas y faunísticas. Asimismo, el abandono intencionado de algunos espacios para posibilitar el proceso natural de sucesión vegetal permite aumentar notablemente la complejidad del hábitat. Sin embargo, este tipo de manejos tendentes a la naturalización de las zonas verdes, admitidos en general por la ciudadanía en los parques periurbanos, suelen generar una fuerte contestación cuando se aplican en las zonas verdes urbanas. En este sentido, es importante el papel que los parques periurbanos pueden jugar en la sensibilización de los ciudadanos respecto a la necesidad de la aplicación de modelos de gestión más respetuosos con la diversidad biológica.

El seguimiento científico de parámetros clave que se ha acometido en el Anillo Verde ha contribuido a criticar objetivamente los resultados obtenidos y reorientar la gestión de estos espacios, aportando en algunos casos argumentos de protección de especies o espacios y facilitando el acceso a algunas vías de financiación.

Por último, la existencia de grandes parques periurbanos con una alta diversidad biológica supone una oportunidad de mejorar la biodiversidad de las zonas verdes en el interior de la ciudad, siempre que sea factible el acondicionamiento de corredores ecológicos a través de la trama urbana. Los viales dotados de anchas medianas ofrecen grandes posibilidades a este respecto.

\section{BIBLIOGRAFÍA}

Agut, A.; 2012. El Jardín Botánico de Olárizu (Vitoria-Gasteiz). Congreso Nacional de Medio Ambiente 2012, Comunicaciones Técnicas. Madrid.

AlCALDE, J.T. y MARTíneZ, I.; 2011. Análisis de la ocupación por murciélagos de los refugios artificiales instalados en el parque de Salburua (Vitoria-Gasteiz). Año 2011. Centro de Estudios Ambientales. Vitoria-Gasteiz.

Alvey, A.A.; 2006. Promoting and preserving biodiversity in the urban forest. Urban Forestry \& Urban Greening 5: 195-201.

BLAIR, R.B.; 1996. Land use and avian species diversity along an urban gradient. Ecol. Appl. 6(2): 506-519. 
BLAIR, R.B.; 1999. Birds and butterflies along an urban gradient: surrogate taxa for assessing biodiversity? Ecol. Appl. 9(9): 164-170.

Celecia, J.; 1997. Urban Ecology: Biodiversity and contemporary stakes of inventories. Journal d'Agriculture Traditionelle et de Botanique Appliquée 39(2): 241-263.

Clark, P.J.; Reed, J.M. \& Chew, F.S.; 2007. Effects of urbanization on butterfly species richness, guild structure, and rarity. Urban Ecosyst. 10: 321-337.

Colding, J.; ElmQvist, T.; Lundberg, J.; Ahrné, K.; Andersson, E.; Barthel, S.; Borgström, S.; Duit, A.; ERnstsson, H. \& TengÖ, M.; 2003. The Stockholm Urban Assessment (SUA-Sweden). Millennium Ecosystem Assessment Sub-Global Summary Report. Stockholm.

Connor, E.F.; HAFernik, J.; Levy, J.; LeE MoOre, V. \& RicKMAN, J.K.; 2002. Insect conservation in an urban biodiversity hotspot: The San Francisco Bay Area. J. Insect Conserv. 6: 247-259.

Cornelis, J. \& Hermy, M., 2004. Biodiversity relationships in urban and suburban parks in Flanders. Landscape and Urban Planning 69: 385-401.

Croci, S.; Butet, A.; Georges, A.; Aguejdad, R. \& Clergeau, P.; 2008. Small urban woodlands as biodiversity conservation hot-spot: a multi-taxon approach. Landscape Ecology 23: 1171-1186.

De La Hera, I.; Unanue, A. y Aguirre, I.; 2009. Efectos del área, edad y cobertura de la vegetación sobre la riqueza de especies de aves reproductoras en los parques urbanos de Vitoria-Gasteiz. Munibe 57: 195-206.

DONOVAN, R.G.; SADLER, J.P. \& BRYSON, J.R.; 2005. Urban biodiversity and sustainable development. Engineering Sustainability 158: 105-114.

Desrochers, A. \& Hannon, S.J.; 1997. Gap crossng decisions by dispersing forest songbirds. Conservation Biology 11: 1204-1210.

FERNÁNDEZ-JURICIC, E.; 2000. Local and regional effects of human disturbance on forest birds in a fragmented landscape. Condor 102: 247-255.

FERNÁNDEZ-JURICIC, E. \& JOKIMÄKI, J.; 2001. A habitat island approach to conserving birds in urban landscapes: case studies from southern and northern Europe. Biodiversity and Conservation 10: 2023-2043.

Gordon, M. \& Forman, R.T.; 1983. Landscape modification and changing ecological characteristics. In: H.A. Money \& M. Godron (eds.), Disturbance and ecosystems: 12-28. Springer-Verlag. New Cork, USA.

GonZÁlEZ, J.A. Y GonZÁlEZ, J.; 2007. El Anillo Verde de Vitoria. Particularidades de la gestión de un parque periurbano. Jardinería Profesional 5: 32-39.

HELDEN, A.J. \& LEATHER, S.R.; 2004. Biodiversity on urban roundabouts-Hemiptera, management and the species-area relationship. Basic and Applied Ecology 5(4): 367-377.

Hohtola, E.; 1978. Differential changes in bird community structure with urbanization: a study in central Finland. Ornis Scandinavica 9: 94-100.

LOBO, L.; 2008. "INBIOS": Incremento de la biodiversidad en los humedales de Salburua.

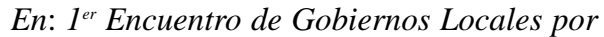
la Biodiversidad. Libro de ponencias: 47-84. FEMP (Federación Española de Municipios y Provincias), Jaén.

MCKINNEY, M.L.; 2002. Urbanization, biodiversity and conservation. BioScience 52: 883890.

MCKINNEY, M.L.; 2006. Urbanization as a major cause of biotic homogenization. Biological Conservation 127: 247-260.

MCKINNEY, M.L.; 2008. Effects of urbanization on species richness: A review of plants and animals. Urban Ecosystems 11: 161-176.

Melles, S.; Glenn, S. \& Martín, K.; 2003. Urban bird diversity and lanscape complexity: Species-environment associations along a multiscale habitat gradient. Conservation Ecology 7(1): 5.

ORTUÑO, V.M. Y MARCOS, J.M.; 1997. Carabidofauna (Insecta, Coleoptera) del área natural de Salburua (Vitoria, Alava). Ecología 11: 457-482.

Pautasso, M.; 2007. Scale dependence of the correlation between human population presence and vertebrate and plant species richness. Ecology Letters 10: 16-24.

Rudd, H.; Vala, J. \& Schaefer, V.; 2002. Importance of backyard habitat in a compre- 
hensive biodiversity conservation strategy: A connectivity analysis of urban green spaces. Restoration Ecology 10(2): 368-375.

Savard, J.-P.l.; Clergeau, P. \& Mennechez, G.; 2000. Biodiversity concepts and urban ecosystems. Landscape and Urban Planning 48: 131-142.

SCHAEFER, V.; 1994. Urban biodiversity. In: E. Harding \& E. McCullum (eds.), Biodiversity in British Columbia. Environment Canada, Canadian Wildlife Service. Vancouver.

Smith, R.M.; Thompson, K.; Hodgson, J.G.; WARREN, P.H. \& GASTON, K.J.; 2006. Urban domestic gardens (IX): Composition and richness of the vascular plant flora, and implications for native biodiversity. Biological Conservation 129: 312-322.
Strohbach, M.W.; HaAse, D. \& Kabisch, N.; 2009. Birds and the city: urban biodiversity, land use, and socioeconomics. Ecology and Society 14(2): 31.

URIBE-ECHEBARRÍA, P.M.; 2010. La flora amenazada del municipio de Vitoria-Gasteiz (Álava). Centro de Estudios Ambientales. Vitoria-Gasteiz.

VALlaDARES, L.F.; 2010. Estudio de la comunidad de Odonatos de los parques del Anillo Verde de Vitoria-Gasteiz. Centro de Estudios Ambientales. Ayuntamiento de VitoriaGasteiz.

Zerbe, S.; Maurer, U.; Schmitz, S. \& Sukopp, H.; 2002. Biodiversity in Berlin and its potencial for nature conservation. Landscape and Urban Planning 944: 1-10. 